\title{
Chronische Niereninsuffizienz erhöht das Schlaganfallrisiko
}

Fragestellung: Sind chronische Nierenerkrankungen bei Patienten mit Vorhofflimmern Prädiktoren für Schlaganfälle und Blutungskomplikationen?

Hintergrund: Vorhofflimmern ist ein wichtiger Risikofaktor für Schlaganfälle, insbesondere bei älteren Menschen. Es gibt eine ganze Reihe von Risikomodellen, um Schlaganfälle und Blutungskomplikationen bei Patienten mit Vorhofflimmern vorherzusagen. Für Schlaganfälle ist der bekannteste Risikoscore der CHADS2-Score, für Blutungskomplikationen der HASBLED-Score [1]. Eine Reihe von Studien zeigt aber, dass offenbar auch chronische Nierenerkrankungen das Risiko von Schlaganfällen und Blutungskomplikationen bei Patienten mit Vorhofflimmern erhöhen.

Patienten und Methodik: Die Arbeitsgruppen aus Dänemark und Großbritannien benutzten das dänische nationale Krankenregister. Identifiziert wurden alle Patienten, die mit der Diagnose Vorhofflimmern zwischen 1997 und 2008 aus dem Krankenhaus entlassen worden waren. Dann wurden Schlaganfälle, systemische Embolien und schwerwiegende Blutungskomplikationen sowie anschließend alle Patienten mit chronischen Nierenerkrankungen und zusätzlich Patienten mit Dialyse oder Nierentransplantation erfasst. Außerdem wurde evaluiert, ob die Patienten Vitamin-K-Antagonisten, Acetylsalicylsäure (ASS) oder eine Kombinationstherapie erhielten. Patienten, die mit Clopidogrel oder Dipyridamol behandelt wurden, wurden von der Studie ausgeschlossen. Studienendpunkte waren Krankenhausaufenthalt oder Tod bedingt durch Schlaganfall oder systemische Thromboembolien, schwerwiegende Blutungen oder Myokardinfarkt sowie Tod jedweder Ursache.

In die Studie wurden 132.372 Patienten mit Vorhofflimmern eingeschlossen. Davon hatten 127.884 (96,6\%) keine Nierenerkrankung, 3.587 eine schwerwiegende Nierenerkrankung und 901 benötigten eine Dialyse oder eine Nierentransplantation. Die Patienten waren im Mittel zwischen 67 und 73 Jahren alt und hatten die für Patienten mit Vorhofflimmern typischen Risikofaktoren. 17-29\% der Patienten erhielten Warfarin, $17-25 \%$ ASS und 5-8,4\% eine Kombinationstherapie.

Ergebnisse: Verglichen mit Patienten mit normaler Nierenfunktion hatten Patienten mit Nierenerkrankungen ein um den Faktor 1,5 erhöhtes Risiko für Schlaganfälle und systemische Embolien. Die Hazard Ratio für Patienten mit Nierenersatztherapie betrug 1,83. Dieser Unterschied war statistisch signifikant. Der Unterschied bestand auch, wenn

Olesen JB, Lip GY, Kamper AL et al. Stroke and bleeding in atrial fibrillation with chronic kidney disease. N Engl I Med 2012; 367: 625-35 Patienten mit transitorischer ischämischer Attacke (TIA) ausgeschlossen wurden. Eine Antikoagulation mit Warfarin reduzierte das Schlaganfallrisiko bei den Patienten ohne Nieren- erkrankung um $40 \%$, bei Patienten mit fortgeschrittener Nierenerkrankung um $15 \%$ und bei Patienten mit Nierenersatztherapie um $56 \%$. ASS war nicht wirksam.

Das Blutungsrisiko war unter Warfarin bei allen beobachteten Patientengruppen um 27 - 36\% erhöht. Eine ähnliche Erhöhung des Risikos von Blutungskomplikationen ergab sich bei ASS.

Schlussfolgerungen: Patienten mit Vorhofflimmern und schwerwiegenden Nierenerkrankungen haben ein deutlich erhöhtes Risiko für Schlaganfälle und schwerwiegende Blutungskomplikationen. Das Schlaganfallrisiko kann signifikant durch eine orale Antikoagulation mit Vitamin-K-Antagonisten reduziert werden, während eine Prophylaxe mit ASS nicht wirksam ist. Der Nutzen der Antikoagulation überwiegt deutlich das Blutungsrisiko.

\section{- Kommentar von Prof. Hans-Christoph Diener Orale Antikoagulation schützt}

Diese wichtige Studie einer großen Patientenpopulation aus Dänemark belegt eindeutig, dass schwerwiegende Nierenerkrankungen ein wichtiger Risikofaktor für Schlaganfälle bei Patienten mit Vorhofflimmern sind. Demgemäß müsste eigentlich eine Niereninsuffizienz in die Risikoscores aufgenommen werden. Wichtig ist die Beobachtung, dass eine orale Antikoagulation das Schlaganfallrisiko hochsignifikant senkt, während Acetylsalicylsäure unwirksam ist. Erwartungsgemäß führt die orale Antikoagulation auch zu einem erhöhten Blutungsrisiko, wobei ähnlich wie bei anderen Populationen mit Vorhofflimmern der Nutzen der Antikoagulation das Blutungsrisiko deutlich übertrifft. Die Ergebnisse sind nicht ohne weiteres auf die neuen Antikoagulanzien zu übertragen, da diese bei schwerwiegenden Nierenfunktionsstörungen kontraindiziert sind.
Referenzen
1. Pisters R et al. Chest 2010; 138: 1093-100

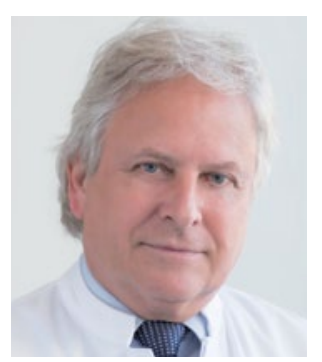

Prof. Dr. med. Hans-Christoph Diener, Essen

Direktor der Klinik für Neurologie, Universitätsklinikum Essen E-Mail:h.diener@uni-essen.de 